\title{
Evaluation du pouvoir fertilisant de deux fumures organiques (fiente de poulet et tourteaux de coprah) sur les paramètres de croissance du bananier plantain (Musa paradisiaca) cultivé sur sables quaternaires du littoral ivoirien.
}

\author{
BAKAYOKO Sidiky ${ }^{1}$, KONATE Zoumana ${ }^{1 *}$, LEKADOU Tacra Thierry², Diomandé Siaka ${ }^{1}$, SORO \\ Dogniméton'. \\ 1Université Jean Lorougnon Guédé de Daloa / Unité de Formation et de Recherche en Agroforesterie. \\ Département : Agropédologie, Télédétection et SIG, BP 150 Daloa (Côte d'Ivoire). \\ ${ }^{2}$ Centre National de Recherche Agronomique (CNRA). 01 BP 1740 Abidjan 01 (Côte d'Ivoire). \\ *Auteur correspondant : e-mail : zoumko@yahoo.fr, Tél. : (00225) 48986475
}

Original submitted in on $8^{\text {th }}$ January 2019. Published online at www.m.elewa.org/journals/ on $28^{\text {th }}$ February 2019 https://dx.doi.org/10.4314/jab.v134i1.10

\section{RESUME}

Objectif : L'objectif de cette étude était d'évaluer le pouvoir fertilisant de deux fumures organiques (fiente de poulet et tourteaux de coprah) sur les paramètres de croissance du bananier plantain (Musa paradisiaca) cultivé sur sables quaternaires du littoral ivoirien.

Méthodologie et résultats : L'étude a été menée à la station de recherche Marc Delorme du Centre National de Recherche Agronomique (CNRA) au Sud de la Côte d'Ivoire sur sables quaternaires du littoral ivoirien avec la variété PITA 3 de bananier plantain avec la fiente de poulet et de tourteaux de coprah. L'essai a été conduit suivant un dispositif expérimental en blocs aléatoires randomisés à trois répétitions. Les plants de bananier de la variété PITA 3 ont été soumis durant dix semaines à huit traitements avec trois traitements à base de fiente de poulet : T2 (500g/plant), T3 $(750 \mathrm{~g} / \mathrm{plant})$ et T4 $(1000 \mathrm{~g} / \mathrm{plant})$, trois traitements à base de tourteaux de coprah : T5 (500g/plant), T6 $(750 \mathrm{~g} / \mathrm{plant})$ et T7 $(1000 \mathrm{~g} / \mathrm{plant})$, un traitement à base d'engrais chimique : T1 (35 g/plant d'urée + 40g/plant de chlorure de potassium) et un traitement témoin (sans apport de fertilisants). . Les observations ont porté sur la circonférence au collet, la taille des plants de bananiers, la surface foliaire et le nombre moyen de feuilles vivantes. Les résultats obtenus ont montré que sur les sables quaternaires du littoral ivoirien, les plants de bananiers traités avec $750 \mathrm{~g} /$ plant de fiente de poulet ou de tourteaux de coprah ont généré les meilleures croissances. Cependant, la fiente de poulet à la dose de $750 \mathrm{~g} /$ plant a permis d'obtenir les meilleures valeurs moyennes de nombre de hauteur des plants $(58,24+9,26)$, de circonférence au collet $(17,119+2,77)$, de surface foliaire $(1534,452 \mathrm{~cm} 2)$ et de feuilles vivantes $(9,16+1,46)$ au cours de l'étude.

Conclusion et application : Sur les sols sableux du littoral, la fertilisation des sols en culture du bananier plantain peut être assurée convenablement par une fertilisation à base de fiente de poulet relativement accessible pour les producteurs généralement à revenus économiques très faibles, à la dose de 750 $\mathrm{g} /$ plant pour assurer la bonne croissance des plants pour une culture de bananier plantain réussie durable, 
Bakayoko et al., J. Appl. Biosci. 2019 Evaluation du pouvoir fertilisant de fumures organiques (fiente de poulet et tourteaux de coprah) sur les de croissance du bananier plantain cultivé sur sables quaternaires du littoral ivoirien.

respectant l'environnement. Toutefois à défaut de la fiente de poulet, le tourteau de coprah également à la dose de $750 \mathrm{~g} /$ plant, pourrait constituer une alternative pour la fertilisation des sols en culture de bananier plantain sur sables quaternaires du littoral ivoirien.

Mots-clés : Banane (Musa spp.), fiente de poulet, tourteaux de coprah, sols sableux, engrais chimiques, Côte d'Ivoire.

\title{
Evaluation of the fertilizing power of two organic fertilizers (chicken dung and coprah meal) on the growth parameters of the plantain banana tree (Musa paradisiaca) grown on quaternary sands of the Ivorian coast.
}

\begin{abstract}
Objective: The objective of this study was to assess the fertilizing properties of two organic fertilizers (chicken droppings and coprah meal) on the growth parameters of the plantain banana tree (Musa paradisiaca) grown on quaternary sands of the Ivorian coast.

Methodology and results: The study was conducted at the Marc Delorme research station of the National Centre for Agronomic Research (CNRA) in southern Côte d'Ivoire on the quaternary sands of the Ivorian coast with the PITA 3 variety of plantain banana tree with chicken droppings and coprah meal. The test was conducted using an experimental device in randomized random blocks with three replicates. Banana tree plants of PITA 3 have been subjected to eight treatments for ten weeks with three chicken dung treatments: T2 (500g/plant), T3 (750g/plant) and T4 (1000g/plant), three coprah meal treatments: T5 (500g/plant), T6 (750g/plant) and T7 (1000g/plant) and a control treatment (without fertilizers). Observations included collar circumference, banana plant size, leaf surface and mean number of live leaves. The results obtained showed that on the quaternary sands of the Ivorian coast, banana plants treated with $750 \mathrm{~g} / \mathrm{plant}$ of chicken droppings or coprah meal have generated the best growth. However, chicken dung at the $750 \mathrm{~g} / \mathrm{plant}$ dose yielded the best mean values of plant height $(58.24+9.26)$, collar circumference $(17.119+2.77)$, foliar surface $(1534.452 \mathrm{~cm} 2)$ and living leaves $(9.16+1.46)$ during the study.

Conclusion and application: Soil fertilization in plantain banana cultivation can be adequately ensured in coastal sandy soils by fertilization based on chicken droppings that are relatively accessible to producers with generally very low economic incomes, at a dose of $750 \mathrm{~g} /$ plant to ensure the proper growth of plants for a successful, sustainable plantain cultivation that respects the environment. However, in the absence of chicken droppings, the coprah meal also at the dose of $750 \mathrm{~g} / \mathrm{plant}$, could be an alternative for fertilization of soils in plantain banana tree culture on the quaternary sands of the Ivorian coast.
\end{abstract}

Keywords: Banana (Musa spp.), Chicken manure, coconut cake, sandy soils, chemical fertilizers, Côte d'Ivoire.

\section{INTRODUCTION}

La banane (Musa spp.) originaire d'Asie du SudEst, est cultivée dans plus de 120 pays du monde (Jones, 2000) où elle joue un rôle important dans la sécurité alimentaire. Elle occupe le quatrième rang des denrées alimentaires les plus importantes après le riz, le blé et le lait dans le monde (FAO, 2010). La banane constitue la principale source alimentaire de nombreuses populations à travers le monde et particulièrement dans les pays tropicaux (Lassois et al., 2009). En Côte d'Ivoire, la culture de la banane occupe le troisième rang parmi les cultures vivrières en termes de tonnages après l'igname et le manioc avec une production moyenne annuelle en 2016 estimée à environ 1,6 millions de tonnes (FAOstat, 2017). Sa culture constitue une source de revenus importants, d'emplois et de recette d'exportation (Foure et Tezenas, 2000). Cependant, en Côte d'Ivoire, la culture de la banane est confrontée à de nombreuses contraintes. En effet, sa culture, pratiquée en majorité par des petits exploitants ruraux à faibles revenus (FAO, 2003) repose 

tourteaux de coprah) sur les de croissance du bananier plantain cultivé sur sables quaternaires du littoral ivoirien.

encore largement sur les petites exploitations de subsistance à faible productivité. Aussi, avec l'accroissement démographique qui entraîne une forte pression sur les réserves foncières, tous les types de sols sont-ils mis en culture (FAO, 2007); c'est le cas des sols sableux de la zone côtière qui connaissent de nos jours une expansion de la culture de la banane. De plus, ces sols sableux sont dotés d'une très grande perméabilité à l'eau et possèdent une très faible fertilité naturelle (Lékadou et al., 2008). Pour remédier à la faible fertilité naturelle de ces sols, l'utilisation des engrais chimiques qui permet de corriger le déficit du sol en élément minéraux et d'améliorer la productivité des cultures (Diallo, 2002) a été envisagée. Toutefois, le coût élevé de ces fertilisants chimiques les rend presque inaccessibles aux petits paysans (Useni et al., 2012). De plus, l'appauvrissement des sols, la destruction de certaines microflores et microfaunes

\section{MATERIEL ET METHODES}

Description du site d'étude : L'étude a été conduite à la station de recherche Marc Delorme du Centre National de Recherche Agronomique (CNRA) au Sud de la Côte d'Ivoire entre $5^{\circ} 14^{\prime}$ et $5^{\circ} 15^{\prime}$ de latitude Nord et $3^{\circ} 54^{\prime}$ et $3^{\circ} 55^{\prime}$ de longitude Ouest (CCT, 2006). Le climat est de type équatorial, caractérisé par quatre saisons (deux saisons humides et deux saisons sèches). La pluviométrie est caractérisée par une précipitation moyenne annuelle de 1673,99 $\mathrm{mm}$. Les températures moyennes mensuelles varient entre 24,5 du sol ainsi que la diminution des teneurs en matières organiques des sols suite à l'utilisation exagérée de ces produits chimiques, limite leur utilisation (Bado, 2002). Cette situation ne permet donc pas de maintenir la fertilité des sols à long terme. Face à cette situation, les agriculteurs utilisent les fertilisants organiques qui constituent une source optimale d'éléments nutritifs pour les plantes (Mulaji, 2011), comme une des solutions alternatives. Cependant, très peu d'études ont été conduites avec les tourteaux de coprah et la fiente de poulet qui sont des fertilisants organiques à moindre coût, disponibles et accessibles pour la plupart des producteurs. La présente étude a été initiée afin d'évaluer le pouvoir fertilisant de deux fumures organiques (fiente de poulet et tourteaux de coprah) sur les paramètres de croissance du bananier plantain (Musa paradisiaca) cultivé sur sables quaternaires du littoral ivoirien

et $27,7^{\circ} \mathrm{C}$ (Lekadou et al., 2008). La végétation qui était constituée de forêt naturelle dense et sempervirente est, cependant, transformée par endroits en plantations de cocotiers et de cultures vivrières. Les sols sont meubles, profonds et aérés (Zakra, 1997) et essentiellement constitués de sables quaternaires homogènes avec une faible teneur en matières organiques et un pH moyen de 5,7 (N'goran, 2005). Les caractéristiques chimiques de ces sols sont présentées dans le tableau 1 ci-dessous.

Tableau 1: Quelques caractéristiques chimiques des sols du site d'étude

\begin{tabular}{c|c|c|c|c|c|}
\hline $\mathrm{pH}_{\text {eau }}$ & $\mathrm{C}(\%)$ & $\mathrm{N}(\%)$ & $\mathrm{CEC}\left(\mathrm{cmol}^{+} \mathrm{kg}^{-1}\right)$ & $\mathrm{SBE}\left(\mathrm{cmol}^{+} \mathrm{kg}^{-1}\right)$ & Pass. $\left(\mathrm{mg}^{-1} \mathrm{~kg}^{-1}\right)$ \\
\hline 5,7 & 0,52 & 0,05 & 4,2 & 0,25 & 42,2 \\
\hline
\end{tabular}
C: : Carbone ; N : Azote ; CEC : Capacité d'échange cationique ; SBE : Somme des bases échangeables ; Pass. : Phosphore
assimilable.

Matériel végétal: Le matériel végétal utilisé était constitué de vivoplants de bananier plantain de la variété améliorée PITA 3. Cette variété est originaire de l'Institut International d'Agriculture Tropicale (IITA) du Nigeria et est tolérante à la maladie des raies noires. Les caractéristiques de la variété PITA 3 sont présentées dans le tableau 2 (CNRA, 2017).

Mise en place de l'essai et dispositif expérimental : L'étude a été réalisée sur une superficie de $2800 \mathrm{~m}^{2}$ (56 $\mathrm{m} \times 50 \mathrm{~m})$. Le dispositif expérimental était constitué de blocs aléatoires randomisés à 3 répétitions (Figure 1). La densité de plantation était de $3 \mathrm{~m}$ entre les lignes et $2 \mathrm{~m}$ entre les plants de bananiers sur la même ligne. Avant la plantation, les plants ont été calibrés afin d'obtenir des plants vigoureux ayant des caractéristiques rapprochées. Les traitements étaient composés en plus du témoin T0 (sans apport de fertilisants), des traitements T2, T3 et T4, constituées 

tourteaux de coprah) sur les de croissance du bananier plantain cultivé sur sables quaternaires du littoral ivoirien.

respectivement de 500,750 et $1000 \mathrm{~g} / \mathrm{plant}$ de fiente de poulet et de T5, T6 et T7, comprenant respectivement 500,750 et $1000 \mathrm{~g} /$ plant de tourteaux de coprah. Le traitement T1 était constitué d'engrais chimiques (Urée : $35 \mathrm{~g} / \mathrm{plant}$; Chlorure de potassium : 40g/plant). Les fertilisants ont été apportés en couronne autour de 20 plants de bananiers constituant la parcelle élémentaire à la dose unique un mois après plantation. Les différentes mesures ont été effectuées sur 4 plants constituant la parcelle utile et les autres plants (16 plants) ont représenté la parcelle de bordure. Ces plants de bordure ont été laissés pour éviter ou réduire les effets de bordures.

Tableau 2 : Caractéristiques agronomiques, technologiques et nutritionnelles de la variété PITA 3.

\begin{tabular}{|l|l|}
\hline Caractéristiques agronomiques & Caractéristiques technologiques et nutritionnelles \\
\hline Cycle 10 mois (cycle court) & Forme du fruit : courbe \\
Intervalle plantation-floraison : 7 mois & Couleur du fruit à maturité : Jaune \\
Intervalle floraison-coupe $: 3$ mois & Texture du fruit : Ferme \\
Hauteur $(\mathrm{m}): 3,367$ & Couleur de la chair à maturité : Orange \\
Nombre de mains par régime : 6.1 & Conservation à l'état vert ou sous forme de \\
Nombre de doigts par régime : 56,6 & farine \\
Longueur de fruit: $21 \mathrm{~cm}$ & \\
$\begin{array}{l}\text { Poids du régime }: 15-18 \mathrm{~kg} \\
\text { Bonne résistance à la cercosporiose }\end{array}$ & \\
Bonne résistance à la fusariose & \\
\hline Rendement & Valorisation \\
Rendement en station : 30 t/ha & Adapté aux mets locaux : alloco, foutou, \\
Rendement en milieu paysan : 20 t/ha & foufou, chips etc... \\
\hline Atout & Faiblesse \\
\hline Haut rendement et résistante à la & Sensible aux nématodes Radopholus similis \\
cercosporiose & et Pratylenchus coffeae \\
\hline
\end{tabular}

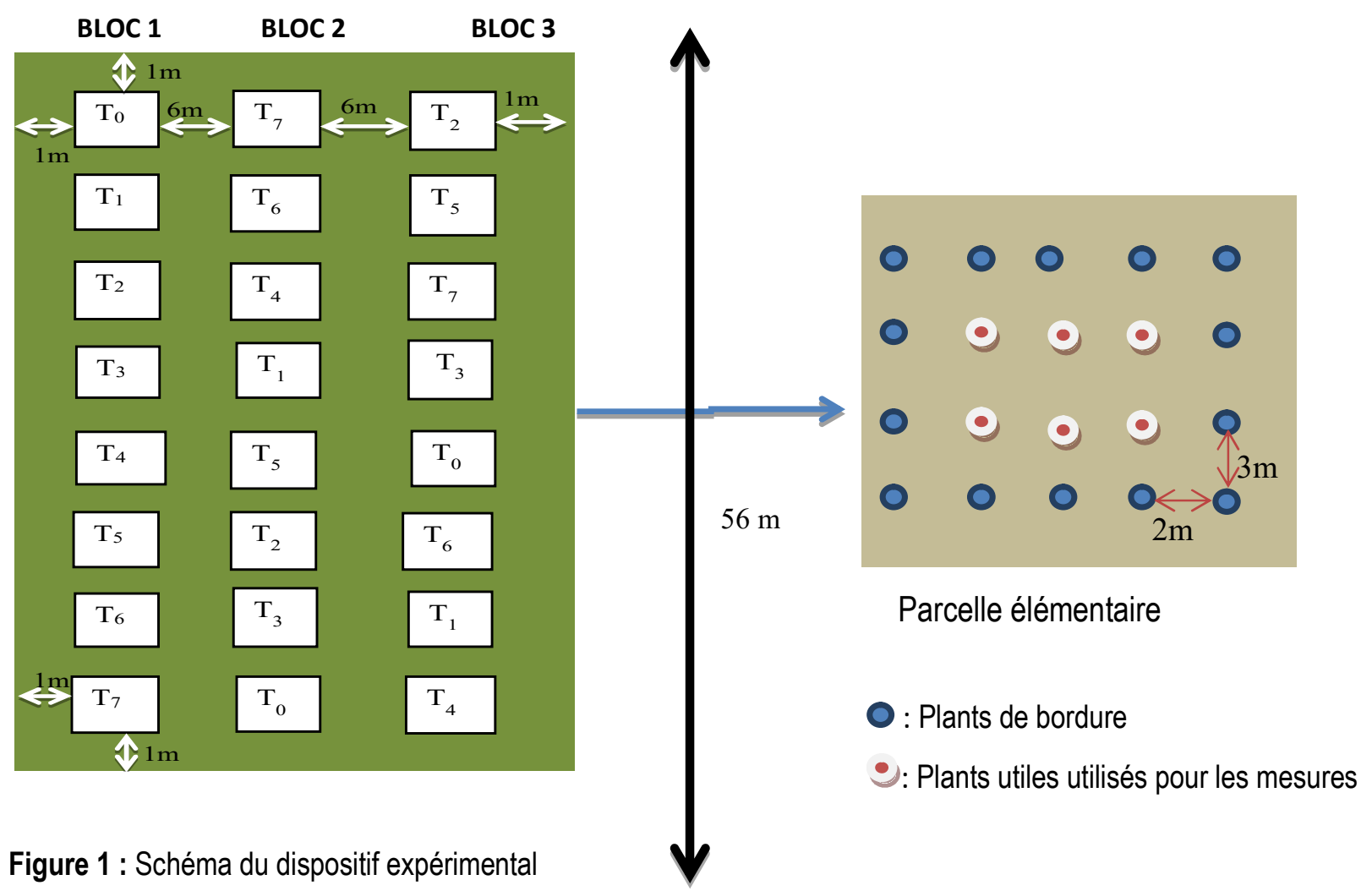



tourteaux de coprah) sur les de croissance du bananier plantain cultivé sur sables quaternaires du littoral ivoirien.

Mesures effectuées au cours de l'expérimentation : Les mesures effectuées concernaient l'évolution de la circonférence au collet, la taille des plants de bananiers, la surface foliaire et le nombre moyen de feuilles vivantes. Toutes ces mesures ont été faites après la mise en place des traitements de la semaine 2 (S2) à la semaine 10 (S10) à raison de deux semaines d'intervalle.

- $\quad$ Mesure de la circonférence au collet: La circonférence au collet $(C)$, mesurée à $5 \mathrm{~cm}$ du sol à l'aide de pied à coulisse a été donnée par la formule suivante : $C=3,14 \times D ; D$ : diamètre au collet et 3,14 , une constante.

- $\quad$ Mesure de la surface foliaire: Elle a été déterminée selon la formule : $S F=0,7 \times L \times \mid+0,23$.

\section{RESULTATS}

Effets des traitements sur la taille des bananiers: Tous les fertilisants ont entraîné un développement significatif de la taille des plants dans le temps (Tableau 3). Les tailles moyennes ont varié d'un fertilisant à un autre et d'un traitement à un autre. Les plus faibles valeurs ont été enregistrées par le traitement T0 (témoin), quel que soit la semaine d'observation. En effet, ces valeurs ont varié entre 22,5 et $26,38 \mathrm{~cm}$. Les valeurs moyennes les plus élevées ont été enregistrées au niveau du traitement $\mathrm{T} 3$ avec la fiente de poulet de la semaine $2(28,30 \mathrm{~cm})$ à la semaine $10(58,24 \mathrm{~cm})$. Par contre, au niveau des tourteaux de coprah, les valeurs les plus élevées ont été enregistrées de la semaine $2(28,68 \mathrm{~cm})$ à la semaine $10(52,36 \mathrm{~cm})$ avec le traitement $\mathrm{T} 6$.
La longueur (L) et la largeur (I) des feuilles ont été mesurées à l'aide d'une règle graduée; 0,7 et 0,23 étant des constantes.

- Mesure de la taille des plants $(H)$ et le nombre moyen de feuilles vivantes de bananiers $(N F V)$ : La taille des plants $(H)$ a été mesurée à l'aide d'une règle graduée. S'agissant du nombre de feuilles (NFV), il a été déterminé par comptage à intervalles réguliers de deux semaines.

Analyses statistiques des données: Les données collectées ont été soumises à une analyse de variance (ANOVA) au moyen du logiciel SPSS (version 22.0). Le test de Newman-keuls au seuil de $5 \%$ a été utilisé pour la comparaison des moyennes. Le logiciel Excel (version 2010) a servi à l'établissement des courbes.

Effet des traitements sur la mesure de la circonférence au collet: Les valeurs moyennes des mesures de circonférences au collet des plants de bananier ont présenté dans l'ensemble des différences entre eux suivant le type de traitement à partir de la deuxième semaine d'application (Tableau 4). Quelle que soit la période d'observation (S2, S4, S6, S8 et $\mathrm{S} 10)$, les traitements T3 au niveau de la fiente de poulet et le traitement T6 au niveau des tourteaux de coprah ont enregistré les valeurs moyennes de circonférence au collet les plus élevées. Les plus faibles valeurs ont été enregistrées par le traitement témoin TO.

Tableau 3 : Evolution de la taille $(\mathrm{cm})$ des plants de bananiers en fonction des périodes d'observation et des traitements.

\begin{tabular}{|c|c|c|c|c|c|c|}
\hline Traitements & fertilisants & Semaine 2 & Semaine 4 & Semaine 6 & Semaine 8 & Semaine 10 \\
\hline T0 & Sans intrants & $\begin{array}{l}22,50 \pm 5,36 \\
b\end{array}$ & $23,66 \pm 6,42 a$ & $24,54 \pm 6,58 a$ & $26,38 \pm 8,03 a$ & $\begin{array}{l}24,76 \pm \\
13,25 a\end{array}$ \\
\hline T1 & $\begin{array}{l}\text { Engrais } \\
\text { minéraux }\end{array}$ & $\begin{array}{l}26,13 \pm 6,97 \\
\text { bc }\end{array}$ & $29,70 \pm 9,84 a b$ & $\begin{array}{l}35,97 \pm 12,51 b \\
c\end{array}$ & $42,52 \pm 15,02 b c$ & $45,86 \pm 15,10 b$ \\
\hline T2 & \multirow{3}{*}{$\begin{array}{l}\text { Fiente de } \\
\text { poulets }\end{array}$} & $\begin{array}{l}22,58 \pm 6,55 \\
a\end{array}$ & $27,82 \pm 8,41 a b$ & $33,72 \pm 9,26 b$ & $40,18 \pm 10,82 b$ & $47,36 \pm 10,56 b$ \\
\hline T3 & & $\begin{array}{l}28,30 \pm 6,74 \\
c\end{array}$ & $38,90 \pm 8,56 e$ & $47,26 \pm 8,82 d$ & $55,39 \pm 9,33 d$ & $58,24 \pm 9,26 c$ \\
\hline T4 & & $\begin{array}{l}26,88 \pm 9,18 \\
\text { bc }\end{array}$ & $\begin{array}{l}35,98 \pm 12,66 \mathrm{~cd} \\
\mathrm{e}\end{array}$ & $\begin{array}{l}42,32 \pm 17,02 c \\
d\end{array}$ & $\begin{array}{l}47,53 \pm 20,00 b c \\
d\end{array}$ & $46,06 \pm 23,10 b$ \\
\hline T5 & \multirow{2}{*}{$\begin{array}{l}\text { Tourteau } \\
\text { de coprah }\end{array}$} & $\begin{array}{l}22,04 \pm 7,16 \\
a b\end{array}$ & $31,79 \pm 7,73 \mathrm{bcd}$ & $\begin{array}{l}39,80 \pm 8,21 b c \\
d\end{array}$ & $47,08 \pm 7,97 \mathrm{bcd}$ & $\begin{array}{l}50,29 \pm \\
7,55 \mathrm{bc}\end{array}$ \\
\hline T6 & & $\begin{array}{l}28,68 \pm 7,90 \\
\text { C }\end{array}$ & $37,42 \pm 10,33 \mathrm{de}$ & $\begin{array}{l}44,18 \pm 11,97 \mathrm{c} \\
\mathrm{d}\end{array}$ & $50,80 \pm 14,13 \mathrm{~cd}$ & $\begin{array}{l}52,36 \pm 14,30 b \\
c\end{array}$ \\
\hline
\end{tabular}


Bakayoko et al., J. Appl. Biosci. 2019 Evaluation du pouvoir fertilisant de fumures organiques (fiente de poulet et tourteaux de coprah) sur les de croissance du bananier plantain cultivé sur sables quaternaires du littoral ivoirien.

\begin{tabular}{|l|l|l|l|l|l|l|}
\hline $\mathrm{T7}$ & $\begin{array}{l}24,56 \pm 6,50 \\
\mathrm{abc}\end{array}$ & $31,48 \pm 8,48 \mathrm{bcd}$ & $\begin{array}{l}38,19 \pm 10,01 \mathrm{~b} \\
\mathrm{c}\end{array}$ & $44,70 \pm 12,10 \mathrm{bc}$ & $46,71 \pm 13,06 \mathrm{~b}$ \\
\hline Moyennes & $24,83 \pm 7,04$ & $32,09 \pm 9,05$ & $38,24 \pm 10,54$ & $44,32 \pm 12,17$ & $46,45 \pm 16,49$ \\
\hline Probabilités & 0,001 & 0,001 & 0,001 & 0,001 & 0,001 \\
\hline $\begin{array}{l}\text { Significativit } \\
\text { é }\end{array}$ & $* \star * * *$ & $* * *$ & $* * *$ & $* * *$ \\
\hline
\end{tabular}

Tableau 4: Evolution des mesures de circonférence $(\mathrm{cm})$ au collet des plants de bananier en fonction de la période d'observation et du traitement.

\begin{tabular}{|c|c|c|c|c|c|c|}
\hline Traitements & $\begin{array}{c}\text { Matières } \\
\text { organiques }\end{array}$ & Semaine 2 & Semaine 4 & Semaine 6 & Semaine 8 & Semaine 10 \\
\hline T0 & Sans apport & $\begin{array}{c}5,639 \pm 2,23 \\
a\end{array}$ & $6,418 \pm 2,48$ & $7,121 \pm 2,80$ & $6,895 \pm 3,30$ & $7,184 \pm 4,37$ \\
\hline T1 & $\begin{array}{l}\text { Engrais } \\
\text { minéraux }\end{array}$ & $\begin{array}{c}7,171 \pm 2,52 \\
a\end{array}$ & $9,030 \pm 3,29$ & $11,077 \pm 3,94$ & $12,874 \pm 4,70$ & $\begin{array}{c}13,702 \pm \\
15,18 \\
\end{array}$ \\
\hline $\mathrm{T} 2$ & \multirow[t]{3}{*}{$\begin{array}{l}\text { Fiente de } \\
\text { poulets }\end{array}$} & $\begin{array}{c}5,438 \pm 1,93 \\
a \\
\end{array}$ & $7,699 \pm 2,40$ & $8,917 \pm 2,43$ & $11,793 \pm 3,10$ & $\begin{array}{c}14,167 \pm \\
3,19 \\
\end{array}$ \\
\hline T3 & & $\begin{array}{c}7,988 \pm 2,05 \\
a\end{array}$ & $10,952 \pm 2,55 a$ & $\begin{array}{c}13,866 \pm 2,46 \\
a\end{array}$ & $\begin{array}{c}15,875 \pm 2,79 \\
a\end{array}$ & $\begin{array}{c}17,119 \pm \\
2,77 \mathrm{a}\end{array}$ \\
\hline T4 & & $\begin{array}{c}7,008 \pm 2,53 \\
a\end{array}$ & $9,294 \pm 3,69$ & $11,630 \pm 4,94$ & $12,572 \pm 6,20$ & $\begin{array}{c}12,798 \pm \\
6,67\end{array}$ \\
\hline T5 & \multirow[t]{3}{*}{$\begin{array}{c}\text { Tourteau } \\
\text { de coprah }\end{array}$} & $\begin{array}{c}6,267 \pm 2,06 \\
a\end{array}$ & $9,025 \pm 2,51$ & $10,537 \pm 2,33$ & $13,665 \pm 2,69$ & $\begin{array}{c}15,360 \pm \\
2,39 a\end{array}$ \\
\hline T6 & & $\begin{array}{c}7,787 \pm 2,56 \\
a\end{array}$ & $10,198 \pm 3,17 a$ & $12,811 \pm 3,70$ & $14,406 \pm 4,12$ & $\begin{array}{c}15,635 \pm \\
4,60 \mathrm{a} \\
\end{array}$ \\
\hline $\mathrm{T} 7$ & & $\begin{array}{c}6,493 \pm 2,18 \\
a \\
\end{array}$ & $8,478 \pm 2,80$ & $11,341 \pm 3,41$ & $12,835 \pm 4,13$ & $\begin{array}{c}13,751 \pm \\
4,58 \\
\end{array}$ \\
\hline Moyennes & & $6,724 \pm 2,40$ & $8,887 \pm 3,13$ & $10,913 \pm 3,85$ & $12,614 \pm 4,66$ & $\begin{array}{c}13,626 \pm \\
5,17\end{array}$ \\
\hline Probabilités & & 0,001 & 0,001 & 0,001 & 0,001 & 0,001 \\
\hline Significativité & & $* * *$ & 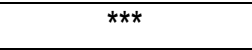 & $* * *$ & $* * *$ & $* * *$ \\
\hline
\end{tabular}

${ }^{\star \star \star}$ : Différence hautement significative

Effet des traitements sur l'évolution de la surface foliaire au cours du temps : L'analyse des données a révélé des différences significatives entre les différents traitements selon les périodes d'observations (Figure 2). Après deux semaines (S2), les valeurs moyennes de surface foliaire les plus faibles ont été obtenues par les traitements T1 $\left(363,498 \mathrm{~cm}^{2}\right)$, T3 $\left(354,484 \mathrm{~cm}^{2}\right)$, T4 $\left(355,528 \mathrm{~cm}^{2}\right)$ et T6 $\left(366,079 \mathrm{~cm}^{2}\right)$. Par contre, les traitements T0 $\left(245,567 \mathrm{~cm}^{2}\right)$, T2 $\left(183,518 \mathrm{~cm}^{2}\right)$, T5 $\left(241,623 \mathrm{~cm}^{2}\right)$ et T7 $\left(251,587 \mathrm{~cm}^{2}\right)$ ont eu les mesures de surface foliaire les plus élevées. Après quatre semaines (S4), les valeurs les plus élevées ont été enregistrées au niveau des traitements T3 $(605,055$ $\left.\mathrm{cm}^{2}\right)$, T4 $\left(533,716 \mathrm{~cm}^{2}\right)$ et T6 $\left(617,686 \mathrm{~cm}^{2}\right)$. Par contre, T0 $\left(279,508 \mathrm{~cm}^{2}\right)$ et T2 $\left(371,413 \mathrm{~cm}^{2}\right)$ ont eu les plus faibles valeurs. Les valeurs intermédiaires étant obtenues par les traitements T1 $\left(451,431 \mathrm{~cm}^{2}\right), T 5$ $\left(459,560 \mathrm{~cm}^{2}\right)$ et $T 7\left(407,825 \mathrm{~cm}^{2}\right)$. Six semaines (S6) après, les traitements T3 $\left(960,924 \mathrm{~cm}^{2}\right)$ et T6 $(890,09$ $\mathrm{cm}^{2}$ ) ont donné les valeurs les plus élevées. Par contre, les plus faibles valeurs étant enregistrées avec le témoin T0 $\left(296,518 \mathrm{~cm}^{2}\right)$. Les traitements T1 $(651,045$ $\left.\mathrm{cm}^{2}\right)$, T2 $\left(590,568 \mathrm{~cm}^{2}\right)$, T4 $\left(799,985 \mathrm{~cm}^{2}\right)$, T5 $(749,605$ $\left.\mathrm{cm}^{2}\right)$ et $\mathrm{T} 7\left(604,488 \mathrm{~cm}^{2}\right)$ ont enregistré les valeurs intermédiaires. Après huit semaines, les traitements T3 $\left(1367,928 \mathrm{~cm}^{2}\right)$ et T6 $\left(1217,806 \mathrm{~cm}^{2}\right)$ ont eu les valeurs les plus élevées. Par contre, le traitement T0 $(359,448$ $\mathrm{cm}^{2}$ ) a eu la plus faible valeur. Dix semaines (S10) après, le traitement T3 $\left(1534,452 \mathrm{~cm}^{2}\right)$ a eu la valeur la 

tourteaux de coprah) sur les de croissance du bananier plantain cultivé sur sables quaternaires du littoral ivoirien.

plus élevée, suivi de T6 $\left(1314,517 \mathrm{~cm}^{2}\right)$ et la plus faible avec le traitement témoin T0 $(7,184 \mathrm{~cm})$.

Evolution du nombre de feuilles vivantes: L'analyse des données a révélé des différences significatives entre les traitements dans le temps $(P<0,001)$ (Tableau 5). Quel que soit le temps d'observation, l'application de la fiente de poulet a permis d'enregistrer les nombres moyens de feuilles vivantes les plus élevés avec le traitement T3 (750 g de fiente par plant). Au niveau des tourteaux de coprah, les doses ont varié également au cours du temps d'observation. En effet, les nombres moyens de feuilles vivantes les plus élevés ont été obtenus avec le traitement T6 $(750 \mathrm{~g}$ de tourteaux de coprah par plant) à la deuxième semaine (6,72 feuilles vivantes) et quatrième semaine $(9,02$ feuilles vivantes). Par contre, à partir de la sixième jusqu'à la dixième semaine, les nombres moyens de feuilles vivantes les plus élevés ont été enregistrés avec le traitement T5 (500 g de tourteaux de coprah par plant).

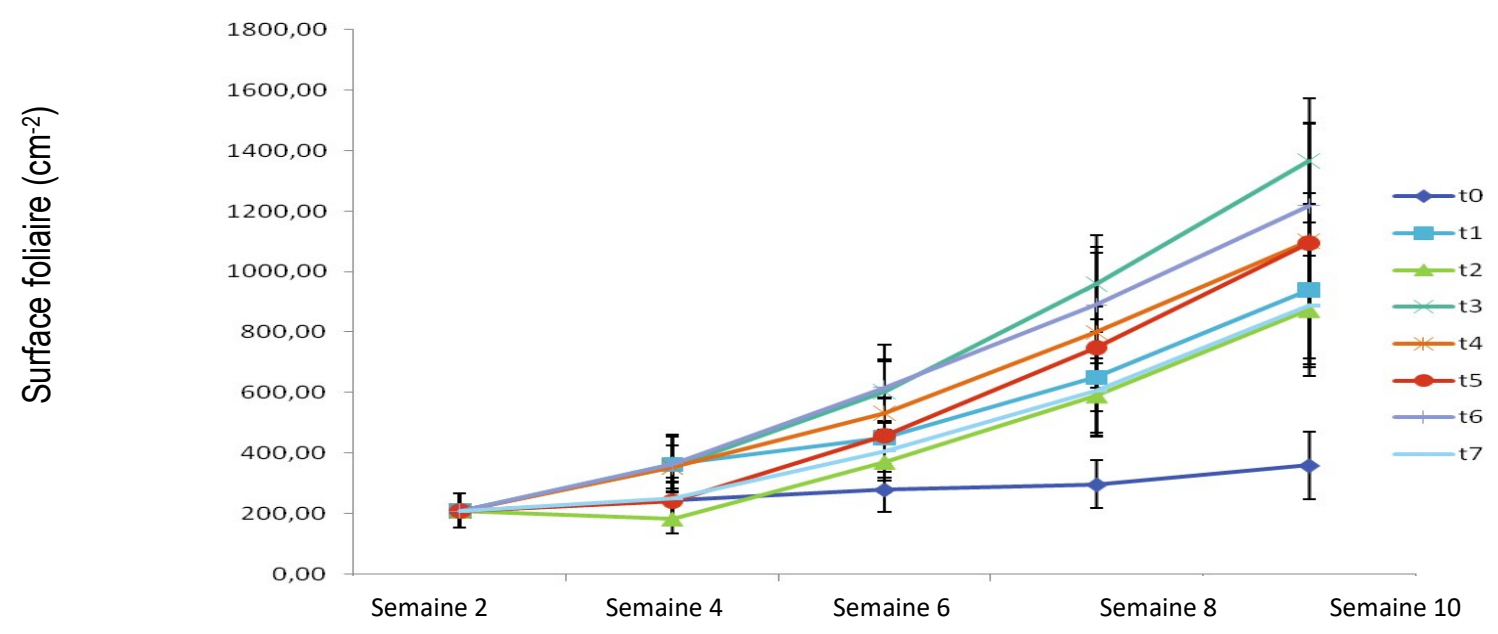

Périodes d'observation (Semaines)

Figure 2 : Evolution de la surface foliaire $\left(\mathrm{cm}^{-2}\right)$ des plants de bananiers traités en fonction de la période d'observation

Tableau 5: Evolution du nombre de feuilles vivantes des plants de bananiers en fonction de la période d'observation et du traitement

\begin{tabular}{|c|c|c|c|c|c|c|}
\hline Traitements & $\begin{array}{l}\text { Matières } \\
\text { organiques }\end{array}$ & Semaine 2 & Semaine 4 & Semaine 6 & Semaine 8 & Semaine 10 \\
\hline T0 & & $4,60 \pm 1,19 a$ & $6,88 \pm 1,13 a$ & $3,32 \pm 1,06 a$ & $2,80 \pm 1,15 a$ & $2,16 \pm 1,21 a$ \\
\hline T1 & & $6,84 \pm 1,74 b c$ & $8,36 \pm 1,80 b c$ & $8,84 \pm 1,95 b$ & $8,44 \pm 2,20 \mathrm{~b}$ & $7,08 \pm 2,28 b$ \\
\hline $\mathrm{T} 2$ & \multirow{3}{*}{ Fiente de poulets } & $6,08 \pm 1,25 b c$ & $8,80 \pm 1,55 b c$ & $\begin{array}{l}9,04 \pm \\
1,33 b c\end{array}$ & $8,76 \pm 1,73 b$ & $8,64 \pm 1,65 c$ \\
\hline T3 & & $7,24 \pm 1,64 c$ & $9,44 \pm 1,19 c$ & $\begin{array}{c}10,20 \pm \\
1,29 c\end{array}$ & $\begin{array}{c}10,16 \pm \\
1,43 b \\
\end{array}$ & $9,16 \pm 1,46 c$ \\
\hline $\mathrm{T} 4$ & & $6,32 \pm 1,74 b c$ & $8,44 \pm 2,00 b c$ & $\begin{array}{c}8,40 \pm \\
2,59 \mathrm{~b}\end{array}$ & $8,40 \pm 3,29 b$ & $7,04 \pm 3,25 b$ \\
\hline T5 & \multirow{3}{*}{$\begin{array}{l}\text { Tourteau } \\
\text { de coprah }\end{array}$} & $6,48 \pm 1,50 \mathrm{bc}$ & $8,84 \pm 167 b c$ & $\begin{array}{l}9,64 \pm \\
1,25 \mathrm{bc}\end{array}$ & $\begin{array}{c}9,16 \pm \\
1,59 \mathrm{~b}\end{array}$ & $8,76 \pm 1,23 c$ \\
\hline T6 & & $6,72 \pm, 51 b c$ & $\begin{array}{l}9,04 \pm \\
1,24 b c\end{array}$ & $\begin{array}{l}9,44 \pm \\
1,38 \mathrm{bc}\end{array}$ & $9,00 \pm 2,17 b$ & $\begin{array}{l}8,24 \pm \\
1,85 \mathrm{bc}\end{array}$ \\
\hline $\mathrm{T} 7$ & & $5,60 \pm 1,68 b$ & $7,80 \pm 1,84 b$ & $8,60 \pm 2,08 b$ & $9,16 \pm 2,44 b$ & $\begin{array}{l}8,12 \pm \\
2,43 \mathrm{bc} \\
\end{array}$ \\
\hline Moyennes & & $6,23 \pm 1,70$ & $8,45 \pm 1,73$ & $8,43 \pm 2,61$ & $8,23 \pm 2,96$ & $7,40 \pm 2,43$ \\
\hline
\end{tabular}


Bakayoko et al., J. Appl. Biosci. 2019 Evaluation du pouvoir fertilisant de fumures organiques (fiente de poulet et tourteaux de coprah) sur les de croissance du bananier plantain cultivé sur sables quaternaires du littoral ivoirien.

\begin{tabular}{|l|c|c|c|c|c|c|}
\hline Probabilités & 0,001 & 0,001 & 0,001 & 0,001 & 0,001 \\
\hline Significativité & & $* * *$ & $* * *$ & $* * *$ & $* * *$ & $* * *$ \\
\hline
\end{tabular}

${ }^{* * *}$ : Différence hautement significative

\section{DISCUSSION}

La variation observée entre le développement des paramètres végétatifs d'un fertilisant à un autre et d'un traitement à un autre serait due non seulement, à l'hétérogénéité des fertilisants et des éléments nutritifs contenus dans ces fertilisants organiques utilisés, mais aussi, à la dose des fertilisants appliqués. Cette variation pourrait également s'expliquer par le degré de décomposition et de minéralisation des fertilisants organiques au moment de leur application, ainsi qu'à la mise à disposition des plants de bananier des éléments nutritifs tels l'azote et le carbone qui au lieu de participer au développement des plants, vont d'abord intervenir dans les processus de décomposition des matières organiques. En effet, pendant la décomposition des matières organiques, l'azote est assimilé dans les protéines cellulaires essentielles pour les fonctions microbiennes. Selon Frey et al., (2003), l'azote contenu dans la matière organique participe à la décomposition de celle-ci et à la croissance des microorganismes décomposeurs. De plus, selon ces auteurs, au premier stade de la décomposition des matières organiques, le carbone est perdu par la respiration et immobilisé dans la biomasse microbienne. Malgré les apports d'eau, le traitement témoin T0 a fourni les faibles valeurs de croissance et de développement des plants de bananiers. Cette situation serait due au manque de fumure qui aurait entraîné un ralentissement, un arrêt ou une absence totale de l'activité des microorganismes du sol qui assurent la décomposition et la minéralisation de la matière organique (Xuluc-Tolosa et al., 2003). L'essai ayant été réalisé sur un sol sableux très pauvre en élément minéraux et en matières organiques, les plants ont été soumis à des conditions où ils étaient obligés de se contenter de la petite quantité d'éléments nutritifs dont disposait ce sol, créant ainsi, des conditions difficiles de développement des plants de bananiers. Ce résultat est soutenu par Lekadou et al., (2008) qui a affirmé que les sols de la zone côtière sont dotés d'une très grande perméabilité à l'eau et d'une très faible fertilité naturelle. Les engrais chimiques (urée et chlorure de potassium) ont positivement influencé la croissance des plantes par rapport aux autres fertilisants deux semaines après application des traitements. Les engrais chimiques ont contribué à l'augmentation de la circonférence au collet et de la surface foliaire deux semaines après son application. Ceci serait du au fait que l'urée est un élément qui contribue à la constitution des feuilles (Ndao, 2009). Aussi, pour la nutrition des plantes, l'urée constitue-t-il une forme d'azote rapidement disponible comme l'ont montré les travaux de Gagnon (2015), qui dans son essai au Québec, a prouvé la rapide assimilation de l'azote par les anguilles. Cependant, l'évolution de ces paramètres avec le traitement à base d'engrais chimique a été ralentie au temps ( $S 4, S 6, S 8$ et $S 10)$ où elles sont restées inférieures à celles des traitements à base de fiente de poulet et de tourteaux de coprah. Ceci serait dû à la nature des sols du site d'étude et à la forte minéralisation de l'engrais apporté. Ces résultats montrent l'action limitée de l'engrais classique qui est soumis aux risques de volatilisation et de lessivage après la pluie, surtout dans les sols sableux (Moughli, 2000). Selon Serpantié et Ouattara (2001), une minéralisation trop rapide favorise les pertes en sels minéraux assimilables et l'acidification du sol, surtout sur les sables tertiaires. Ce qui a donc entraîné un ralentissement du rythme de croissance des plantes traités avec l'engrais chimique classique par rapport à la fiente de poulet et aux tourteaux de coprah. Après la deuxième semaine d'application, ces paramètres ont commencé à être améliorés avec l'application de la fiente de poulet et des tourteaux de coprah. L'amélioration de ces paramètres pourrait s'expliquer par la période d'application de deux semaines qui semble suffisante pour initier l'activité des microorganismes et permettre une décomposition et une minéralisation des résidus organiques. Cette période a permis à ces fertilisants organiques, d'améliorer le niveau des éléments minéraux du sol, nécessaires pour la croissance des plants en particulier l'azote, élément essentiel pour la croissance des plants. L'importance des matières organiques a été signalée par les travaux de Giller et al. (2002) qui ont indiqué que la matière organique est le meilleur fertilisant de base des cultures. Selon Huber et Schaub (2011), la fumure organique joue un rôle important dans l'amélioration de la fertilité des sols à travers leurs actions sur les propriétés physiques, biologiques et chimiques des sols. Ces fertilisants organiques ont 

tourteaux de coprah) sur les de croissance du bananier plantain cultivé sur sables quaternaires du littoral ivoirien.

induit une meilleure croissance des plants par rapport au témoin et aux engrais chimiques durant tout le cycle du bananier. Cette croissance pourrait s'expliquer par la bonne teneur de ces fertilisants organiques en éléments nutritifs notamment l'azote, le phosphore et le potassium et à l'amélioration des propriétés physicochimiques et biologiques des sols. Selon Amidou et al. (2005), l'apport des fertilisants organiques améliore la pénétration des racines, la circulation de l'air et de l'eau dans le sol ainsi que la formation et l'entretien du complexe argilo-humique. Ces résultats corroborent ceux de Weber et al. (2007) et Kitiaba et al. (2016) qui ont affirmé que l'utilisation des matières organiques augmente la fertilité des sols en améliorant leur structure, leur capacité de rétention en eau et en nutriments ainsi que la stimulation de l'activité microbienne du sol. Ces résultats révèlent ainsi, l'importance de ces fertilisants organiques dans la croissance du bananier. La présence de ces fertilisants organiques a ralenti considérablement durant les quatre premières semaines (S2 et S4), la croissance et le développement des plants de bananiers. Au niveau des tourteaux de coprah apportés au sol, ceci pourrait s'expliquer par la nature du fertilisant organique. La présence de lignine dans la constitution des tourteaux de coprah semblerait affecter négativement les facteurs intervenant dans les processus de décomposition de ces matières organiques par les micro-organismes du sol. En effet, la lignine intervient en formant des complexes avec les protéines présentes dans les matières organiques, et les rend résistantes à la minéralisation (Duguet, 2005). Selon Hartemink (2001), les résidus de culture et les amendements organiques, riches en lignine, grâce à leurs propriétés antibiotiques sur les micro-organismes décomposeurs, exercent une action inhibitrice sur la décomposition des résidus. Par contre, au niveau de la fiente de poulet, ceci pourrait s'expliquer par une importante quantité apportée au sol. Dans ces conditions, pour accroître leur nombre et leur activité pour la décomposition de cette quantité de matières organiques, les micro-organismes sont obligés, au cours des premières semaines d'application, de se multiplier en quantité suffisante, en utilisant comme nourriture, les matières organiques et les éléments préexistants, présents dans le sol, tels l'azote, le phosphore et le potassium (Ndiaye, 1999) qui, en réalité devraient servir à la nutrition des plants de bananiers. Selon Feller et al. (2000), la matière organique constitue la base nutritionnelle des plantes et des micro-organismes du sol qui y puisent leur énergie. Cela aurait ainsi crée pendant les premières semaines, au niveau de ces deux fertilisants organiques, une concurrence vis-à-vis des nutriments, entre les plants de bananiers et les micro-organismes du sol. Concernant l'évolution du nombre moyen de feuilles vivantes, le traitement T3 (750 g/plant) à base de fiente de poulet a permis d'obtenir les nombres moyens les plus élevés tout au long du cycle de la banane. Par contre, au niveau des tourteaux de coprah, ces valeurs moyennes ont été améliorées avec le traitement T6 (750 g/plant) jusqu'à la semaine 4 et à partir de la semaine 6 jusqu'à la semaine 10, c'est le traitement T5 (500 g/plant) qui a pris le relais. Cette différence pourrait s'expliquer non seulement, par la vitesse de libération des éléments nutritifs contenus dans ces matières organiques, mais aussi, par leur nature. Au niveau de la fiente de poulet, leur richesse en éléments nutritifs surtout azotés et leur vitesse de libération des nutriments pourrait justifier cette différence. En effet, l'azote est un facteur essentiel de la croissance des plants, surtout au niveau des tiges et des feuilles (Frey et al., 2003). Selon Nyembo et al. (2012), la fiente de poulet présente une meilleure minéralisation, donc libère beaucoup plus vite l'azote pour le mettre à la disposition des plantes. Les travaux réalisés par Pamo et al. (2002), ont montré que la fiente de poulet à une aptitude à enrichir le sol en azote de façon naturelle, faisant de l'utilisation de ces déjections animales, une pratique courante en agriculture et fortement recommandés en agriculture biologique (Reganold et al., 2001). Ces résultats confirment ceux de Demir et al. (2010) et Delago et al. (2012) qui ont montré que le fumier de volailles est un produit très concentré en éléments fertilisants (azote, potasse et phosphore et en oligo-éléments). L'amélioration des paramètres agronomiques avec le traitement T5 (500g/plant) à partir de la semaine 6 serait dû à une lente vitesse de libération des éléments nutritifs contenus dans les tourteaux de coprah durant les 4 semaines qui n'ont pas permis d'améliorer de façon substantielle la fertilité des sols. Ceci pourrait expliquer qu'en 4 semaines, la totalité des tourteaux ne soit pas décomposée. Ce type de minéralisation serait plutôt convenable car selon Serpantié et Ouattara (2001), une minéralisation trop rapide favorise les pertes en sels minéraux assimilables et l'acidification du sol surtout sur les sables tertiaires. 


\section{CONCLUSION}

Les plants de bananiers traités avec $750 \mathrm{~g}$ de fiente de poulet/plant ou $750 \mathrm{~g}$ de tourteaux de coprah/plant ont engendré les meilleurs taux de croissance. Toutefois, les producteurs de bananier plantain sur sables quaternaires du littoral ivoirien gagneraient plus en apportant la fiente de poulet en raison de $750 \mathrm{~g} /$ plant pour assurer la bonne croissance des plants. Toutefois

\section{REMERCIEMENTS}

Les auteurs remercient I'Université Jean Lorougnon Guédé pour leur collaboration et le Centre National de

\section{REFERENCES BIBLIOGRAPHIQUES}

Amidou M., Djènontin AJ., Wennink B., 2005. Valorisation des résidus de récolte dans l'exploitation agricole au nord du Bénin : utilisation du fumier produit dans le parc de stabulation des bœufs. Bulletin de la Recherche Agronomique du Bénin. 47 : 19-25.

Bado B.V., 2002. Rôle des légumineuses sur la fertilité des sols ferrugineux tropicaux des zones guinéenne et soudanienne du Burkina Faso, mémoire de thèse (philosophie), Département des sols et de génie agroalimentaire. Faculté des sciences de l'agriculture et de l'alimentation, Université LAVAL, QUÉBEC, $197 \mathrm{p}$.

CCT, 2006. Centre de Cartographie et de Télédétection du Bureau National d'Etudes Techniques et du Développement (BNETD). Abidjan, Côted'Ivoire, $264 \mathrm{p}$.

CNRA. 2017. Répertoire des variétés améliorées de culture vivrières. Edition 2017, $73 p$.

Delago M., Rodriguez C., Martin JV., De Imperial RM., et Alonso F., 2012. Environmental assay on the effect of poultry manure application on soil organisms in agroecosystems. Science of the total environment, 416: 532-535.

Demir K., Sahin O., Kadioglu YK., Pilbeam DJ. and Gunes A., 2010. Essential and non essential element composition of tomato plants fertilized with poultry manure. Scientia horticulturae, 127 (1) : 16-22.

Diallo L., 2002. Effet de l'urée et du fumier sur le rendement du maïs. Mémoire d'ingénieur du Développement Rural / Option Agronomie. IDR / UPB. Burkina Faso. 54 p.

Duguet F., 2005. Minéralisation de l'azote et du phosphore dans les sols organiques cultivés du Sud-Ouest du Québec, Mémoire présenté à défaut de la fiente de poulet, le tourteau de coprah également à la dose de $750 \mathrm{~g} /$ plant, pourrait constituer une alternative.. II serait donc profitable pour ces producteurs généralement à revenus économiques très faibles, de privilégier l'usage de la fiente de poulet, relativement accessible.

Recherche Agronomique (CNRA) pour son appui financier.

pour l'obtention du grade de maître ès sciences (M.Sc.), Université de Laval (Québec), Faculté des sciences de l'agriculture et de l'alimentation. $105 \mathrm{p}$.

FAO, 2003. Les engrais et leurs applications : Précis à l'usage des agents de vulgarisation agricole, quatrième édition, Rabat 2003, $84 \mathrm{p}$.

FAO, 2007. L'étude prospective du secteur forestier en Afrique (FOSA), rapport FAO, (Sénégal), $22 p$.

$\mathrm{FAO}, 2010$. Les répercussions du changement climatique sur la sécurité alimentaire et la gestion des ressources naturelles en Afrique. Document de référence rédigé pour la vingtsixième Conférence régionale pour l'Afrique. Luanda, Angola, 3-7.

FAOstat, 2017. Division de la statistique de l'Organisation des Nation Unies pour l'Alimentation et l'Agriculture. http://www.fao.org/faostat/fr/\#data/QC.

Feller C., Balesdent J., Nicolardot B. and Cerri C.C., 2000. Approaching "functional" soil organic matter pools through particle-size fractionation. Examples for tropical soils. In: Assessment methods for soil carbon (eds R. Lal, J.M. Kimble, R.F. Follett et B.A. Stewart), pp. 5367. Lewis Publisher, Boca Raton.

Foure E. et Tezenas DM., 2000. Les productions bananières : un enjeu économique majeur pour la sécurité alimentaire. Bulletin de liaison de la coopération régionale pour le développement des produits horticoles en Afrique, 18 : 23-28.

Frey S. D., Elliot E. T., Paustian K. and Peterson G. A., 2003. Fungal translocation as a mechanism for soil nitrogen inputs to surface residue decomposition in a no-tillage agroecosystem. Soil Biol \& Bioch 32: 689-698. 
Giller K.E., Cadisch G. and Palm C., 2002. The NorthSouth divide: Organic wastes or resources of nutrient management. Agronomy 22, pp 703709.

Huber G. et Schaub C., 2011. La fertilité des sols : l'importance de la matière organique. Agriculture et terroir, chambre d'agriculture Bas Rhin, $46 \mathrm{p}$.

Hartemink A.E. and O'Sullivan J.N., 2001. Leaf litter decomposition of Piper aduncum, Glicidia sepium and Imperata cylindrical in the humid lowlands of Papua New Guinea. Plant Soil, 230, 115-124.

Jones D.R., 2000. Diseases of Banana, Abacá and Enset. $\mathrm{CABI}$ Publishing. Wallingford, Oxon, UK. 544 p.

Kitabala MA., Tshala UJ., Kalenda MA., Tshijika IM. et Mufind KM., 2016. Effets de différentes doses de compost sur la production et la rentabilité de la tomate (Lycopersicon esculentum Mill) dans la ville de Kolwezi, Province du Lualaba (RD Congo). J. Appl. Biosci. 102 : 9669-9679. http://dx.DOl.org/10.4314/jab.v102i1.1.

Lassois L., Busogoro J-P. et Jijakli H., 2009. La banane : de son origine à sa commercialisation. Biotechnol. Agron. Soc. Environ., 13 (4) : 575586.

Lekadou T., Alice N., Jean-Louis K., Kouassi A., Zakra $\mathrm{N}$. et ASSA A., 2008. Décomposition des tourteaux de coprah et de palmiste et effets sur la croissance des cocotiers (Cocos nucifera L.) en pépinière et la nutrition minérale des cocotiers adultes en Côte d'Ivoire, Sciences et Nature Vol. $5 N^{\circ} 2: 155$ 166.

Moughli L., 2000. Transfert de technologie : les engrais minéraux caractéristiques et utilisation. Bulletin d'information et de liaison du PNTTA (72), $4 \mathrm{p}$.

Mulaji K., 2011. Utilisation des composts de biodéchets ménagers pour l'amélioration de la fertilité des sols acides de la province de Kinshasa (République Démocratique du Congo). Thèse Université de Liège, Belgique, $191 \mathrm{p}$.

Ndao A., 2009. Cultures maraîchères et dynamiques socio-économiques et spatiales dans la communauté rurale de Ndiob (département de Fatick). Université Gaston Berger de SaintLouis. Mémoire DEA, $147 \mathrm{p}$.

Ndiaye N., 1999. Les nématodes du sol dans les systèmes de culture à jachères du Sénégal :
Dynamique des peuplements et relations avec les facteurs abiotiques et biotiques telluriques.

Thèse de Doctorat de 3ème Cycle de Biologie Animale. Université Cheikh Anta Diop de

Dakar .Soutenu le 5 Février 1999 à la Faculté des Sciences et Techniques. $170 \mathrm{p}$.

N'goran A., 2005. Amélioration de la fertilité chimique des sables quaternaires en Côte d'Ivoire dans l'association cocotier/acacia Spp. Université de Gent, Belgique, Thèse, $193 \mathrm{p}$.

Nyembo KL., Useni SY., Mpundu MM., Bugeme MD., Kasongo LE. et Baboy LL., 2012. Effets des apports des doses variées de fertilisants inorganiques (NPKS et Urée) sur le rendement et la rentabilité économique de nouvelles variétés de Zea mays L. à Lubumbashi, SudEst de la RD Congo. Journal of Applied Biosciences, 59: 4286-4296.

Pamo E., Boukila B., Tonfack L., Momo M., Kana J. et Tendonkeng F., 2002. Influence de la fumure organique, du NPK et du mélange des deux fertilisants sur la croissance de Moringaoleifera dans l'ouest Cameroun. Livestock Research for Rural Development 17 (3): 115-135.

Reganold JP. Glover JD., Andrews PK. and Hinman HR., 2001. Organic farming and the sustainability of agricultural systems. 21- 40 .

Serpantié G. et Ouattara B., 2001. Fertilité et jachères en Afrique de l'Ouest. In : Floret C. \& Pontanier R., Eds. La jachère en Afrique tropicale (volume 2). Paris, France : Jhon Libbey Eurotext. pp 21-83.

Useni S., Baboy L., Nyembo K. et Mpundu M., 2012. Effets des apports combinés de biodéchets et de fertilisants inorganiques sur le rendement de trois variétés de Zea mays L. cultivées dans la région de Lubumbashi. J. Appl. Biosci. $54: 3935-3943$.

Weber J., Karczewska A., Drozd J., Lieznar M., Lieznar S., Jamroz E. and Kocowiez A., 2007. Agricultural and ecological aspects of sandy soil as affected by the application of municipal solid waste composts. Soil Biol. Biochem. 39: 1294-1302.

Xuluc-Tosola F.J., Vester H.F.M., Ramirez-Marcial N., Castellanos-Albores J. and Lawrence D., 2003. Leaf litter decomposition of the tree species in three successional phases of tropical dry secondary forest in Campeche, Mexico. For Ecol and Man 174: 401-412. 
Bakayoko et al., J. Appl. Biosci. 2019 Evaluation du pouvoir fertilisant de fumures organiques (fiente de poulet et tourteaux de coprah) sur les de croissance du bananier plantain cultivé sur sables quaternaires du littoral ivoirien.

Zakra N., 1997. Contribution à l'étude de la restauration et au maintien de la fertilité des sables quaternaires du littoral ivoirien: cas de l'utilisation d'arbres fixateurs biologiques d'Azote comme plante associative avec les cocotiers. Thèse, Docteur-ingénieur, Université de Cocody (Côte d'Ivoire), $155 \mathrm{p}$. 